\title{
Riscos psicossociais associados à síndrome de burnout em professores universitários
}

\section{Psychosocial Risks fssociated with Burnout Syndrome fmong University Professors Riesgos psicosociales asociados con el síndrome de burnout en profesores universitarios}

\author{
Mary Sandra Carlotto* \\ Universidade do Vale do Rio dos Sinos \\ Sheila Gonçalves Câmara* \\ Universidade Federal de Ciências da Saúde de Porto Alegre - UFCSPA
}

Doi: http://dx.doi.org/10.12804/revistas.urosario.edu.co/apl/a.4036

\section{Resumo}

A síndrome de burnout é um fenômeno psicossocial que surge como uma resposta crônica aos estressores psicossociais presentes no contexto de trabalho, sendo constituída por quatro dimensões: Ilusão pelo Trabalho, Desgaste Psicológico, Indolência e Culpa. O objetivo deste estudo foi avaliar os estressores ocupacionais de natureza psicossocial como possíveis preditores das dimensões da síndrome de burnout em uma amostra de 250 professores universitários. Como instrumento de avaliação foi utilizado o Questionário para Avaliação da Síndrome de Burnout (Cuestionario para la evaluación del sindrome de burnout por el Trabajo - CESQT-Ed) e a Bateria de riscos psicossociais. Os resultados, obtidos por meio da análise de regressão linear, apontaram as variáveis "Autonomia”, "Apoio social”, "Conflito de papel" e "Conflitos interpessoais" como principais preditores de burnout. O estudo sugere possíveis intervenções e possibilidades de novos estudos.

Palavras-chave: síndrome de burnout; Risco psicossocial no trabalho, Professores universitários.

\section{fibstract}

Burnout syndrome is a phenomenon that emerges as a chronic response to psychosocial stressors in the work environment. It has four dimensions: enthusiasm for the job, psychological exhaustion, lassitude, and guilt. The objective of this study was to evaluate the psychosocial stressors related to work as possible predictors of dimensions of burnout syndrome among a sample of 250 university professors. The Spanish Burnout Inventory - Educational Version (SBI-Ed) and the Battery of Psychosocial Risk Assessment were used as research instruments. Results obtained through linear regression

* Correspondência: Programa de Pós-Graduação em Psicologia, Escola de Saúde - Sala E01-118. Universidade do Vale do Rio dos Sinos. Av. Unisinos, 950 - Cristo Rei, CEP: 93.022-750 - São Leopoldo - RS/Brasil. Correio eletrônico: mscarlotto@gmail. com

Cómo citar este artículo: Carlotto, M. S., \& Câmara, S. G. (2017). Riscos psicossociais associados à síndrome de burnout em professores universitários. Avances en Psicología Latinoamericana, 35(3), 447-457. doi: http://dx.doi.org/10.12804/10.12804/ revistas.urosario.edu.co/apl/a.4036 
analysis point to the variables of autonomy, social support, role ambiguity, and interpersonal conflicts as predictors of burnout. The study suggests possibilities for intervention and further research.

Keywords: Burnout syndrome, psychosocial risk at work, university professors.

\section{Resumen}

El síndrome de burnout es un fenómeno psicosocial que ocurre como respuesta crónica a los estresores psicosociales presentes en el contexto del trabajo. Constituyese de cuatro dimensiones, Ilusión por el trabajo, Desgaste Psicológico, Indolencia y Culpa. El objetivo de este estudio fue evaluar los estresores ocupacionales de naturaleza psicosocial, como posibles predictores de las dimensiones del síndrome de burnout en una muestra de 250 profesores universitarios. Como instrumento de evaluación fue utilizado el Cuestionario para la Evaluación del síndrome de burnout por el Trabajo (CESQT) y la Batería de riesgos psicosociales. Los resultados, obtenidos por medio del análisis de regresión lineal apuntaron a las variables "autonomía", "apoyo social", "conflicto de papel" y "conflictos interpersonales" como principales predictoras de burnout. El estudio sugestiona posibles intervenciones y posibilidades de nuevos estudios.

Palabras clave: síndrome de burnout, riesgos psicosociales en el trabajo, profesores universitarios.

\section{Introdução}

A síndrome de burnout (SB) é um fenômeno psicossocial que surge como uma resposta crônica aos estressores presentes no contex to de trabalho (Maslach, Schaufeli \& Leiter, 2001; Leiter, Bakker, \& Maslach, 2014). Na perspectiva psicossocial, a SB é um tipo particular de mecanismo de enfrentamento e de autoproteção frente ao estresse gerado, principalmente na relação estabelecida entre prestadores e receptores de serviços (Gil-Monte, 2008). Estressores psicossociais quando persistentes podem ocasionar a SB (Guglielmi \& Tatrow, 1998; Norlund, Reuterwall, Höög, Janlert \& Järvholm, 2015).

No modelo desenvolvido por Gil-Monte (2005), a SB se constitui de quatro dimensões: 1. Ilusão pelo Trabalho, definida como o desejo do trabalhador para alcançar as metas de trabalho, uma vez que supõe ser este uma fonte de prazer pessoal e realização profissional; 2. Desgaste Psíquico, dimensão caracterizada pelo surgimento de esgotamento emocional e físico, decorrente do fato de ter que lidar cotidianamente em seu trabalho com pessoas que apresentam ou causam algum tipo de problema; 3. Indolência, definida como a presença de atitudes negativas de indiferença, insensibilidade e distanciamento frente às pessoas com as quais necessita se relacionar no contexto de trabalho; 4. Culpa, entendida pelo surgimento de sentimentos de culpabilização por atitudes e comportamentos não condizentes com as normas internas e cobrança social acerca do papel profissional.

O docente está exposto a diversos estressores ocupacionais que o tornam vulnerável à SB. Estressores ocupacionais são aspectos do trabalho, que geram tensão decorrente do desequilibro entre a percepção das demandas de trabalho, como um estressor e a avaliação por parte do trabalhador sobre sua capacidade em termos de conhecimentos e habilidades para manejá-lo adequadamente (Leka, Griffiths \& Cox, 2003). Os estressores ocupacionais psicossociais são definidos por Cooper, Dewe \& O'Driscoll (2001) como aqueles relacionados à natureza do trabalho e relações interpessoais.

A sB é um fenômeno que atinge os professores de diferentes países, tendo um caráter epidêmico mundial, que extrapola as fronteiras nacionais (Gil-Monte, 2008; Gupta \& Rani, 2014). Professores, no entanto, formam um grupo heterogêneo, pois dentro desta categorização há diferenças nas funções, atribuições, desafios e recompensas, dependendo do nível de ensino em que atuam e contexto social no qual as instituições estão inseridas (Guglielmi \& Tatrow, 1998). 
No caso da docência em nível de ensino superior, essa tem sido caracterizada como uma atividade complexa do ponto de vista político, social, intelectual, psicológico e pedagógico, que se realiza no eixo formado pelo tripé ensino-pesquisa-extensão (Franco, 2001; Minter, 2009). Suas atividades envolvem competências cognitivas, funcionais, comportamentais, éticas e políticas (Mendonça, Paiva, Padilha, \& Barbosa, 2012). A diversidade e a complexidade presentes no atual ambiente acadêmico decorrem fundamentalmente das influências das políticas educacionais e das relações entre a universidade e a sociedade. Essa dinâmica atinge a estrutura e o funcionamento da universidade e o trabalho docente (Silva, 2011).

Professores universitários constituem uma categoria laboral com algumas especificidades, pois trabalham com atividades acadêmicas, de investigação e de gestão em uma proporção muito variada. Por um lado, embora tenha um componente de produtividade controlada como outras profissões, suas atividades se diferenciam pelo caráter de auto-organização e pela alta motivação intrínseca (Moreno-Jiménez, Garrosa, Rodríguez, Martínez \& Ferrer, 2010). A atividade docente também tem sofrido um processo de corrosão das suas condições de trabalho nas últimas três décadas (Barkhuizen \& Rothmann, 2008). Os salários foram defasados, os contratos de trabalho flexibilizados, a carga de trabalho aumentada e o número de níveis de ascensão profissional diminuíram ou estagnaram, a pressão para obter financiamento externo aumentou e tem sido colocada maior ênfase para a realização de publicação (Catano et al., 2010).

Além disso, o professor vem perdendo a autonomia sobre suas tarefas, é controlado nas suas atividades, trabalha sob forte pressão, com pouco reconhecimento, recompensas profissionais e possibilidades de promoção lentas e limitadas (Gillespie, Walsh, Winefield, Dua \& Stough, 2001; Kang \& Sidhu, 2015; Tytherleigh, Webb, Cooper $\&$ Ricketts, 2005), tendo que atender este aumento de demanda com recursos materiais insuficientes e pouco apoio social (Moreno-Jiménez et al. 2010; Salami, 2011).

O trabalho sob essas condições faz com que o professor gradualmente desenvolva sentimentos de inadequação no desempenho de suas tarefas e incapacidade de lidar com os estressores, gerando sentimentos de exaustão emocional (Tümkaya, 2006). A quantidade e a gravidade dos estressores ocupacionais por um longo período de tempo podem conduzir à SB, resultando em comportamentos disfuncionais e comprometendo o desempenho e a qualidade da atividade docente (Guglielmi \& Tatrow, 1998; Minter, 2009). Outra importante consequência é o pensamento de abandonar a profissão (Moreno-Jiménez et al., 2010; Minter, 2009). Estressores ocupacionais têm desempenhado um papel central na previsão das dimensões da SB em professores universitários (Salami, 2011). Dentre os estressores ocupacionais associados à SB em professores, estudos têm destacado o conflito de papel (Farber, 1991; Papastylianou, Kaila \& Polychronopoulos, 2009), a falta de autonomia (Yong \& Yue, 2007), a sobrecarga laboral, a presença de conflitos interpessoais no trabalho e o baixo apoio social (Droogenbroeck, Spruyt \& Vanroelen, 2014). Vários estudos sobre SB têm sido desenvolvidos com professores, mas ainda é proporcionalmente pequena à quantidade de estudos desenvolvidos especificamente com professores universitários (Minter, 2009). Assim, pelo exposto, o estudo teve como objetivo avaliar os estressores ocupacionais de natureza psicossocial, como possíveis preditores das dimensões da SB em professores universitários.

\section{Método}

\section{Participantes}

Este estudo de corte transversal teve como amostra não probabilística, 250 professores universitários de duas instituições de ensino privado da região metropolitana de Porto Alegre. Foram 
incluídos somente professores que atuavam no ensino superior há mais de um ano.

Segundo o sexo, $51,6 \%$ dos sujeitos se identificam como mulheres e 48,4\% como homens. A média de idade ficou entre 41 e 45 anos (Desvio-padrão, $\mathrm{DP}=11,25)$. Com relação à situação conjugal, $82,5 \%$ referem ter uma união estável e $79,2 \%$ possuem filhos. A maioria dos participantes possui formação em nível de pós-graduação $(97,5 \%)$ e trabalha em uma única instituição (66,5\%). A média de tempo de experiência profissional foi de 9,47 anos (DP $=8,42)$ e a média de alunos atendidos diariamente foi de 91 (DP = 24). A carga horária de trabalho semanal variou de 12 a 52 horas, sendo a média de 30,8 ( DP $=8,5)$.

\section{Instrumentos}

Os dados foram coletados por meio dos seguintes instrumentos autoaplicáveis: 1. Questionário de dados sociodemográficos (sexo; idade; relação conjugal; filhos) e laborais (carga horária; tempo de exercício no ensino superior; número de alunos atendidos diariamente; trabalhar em outra instituição); 2. Questionário para avaliação da síndrome de burnout, versão para professores - Cuestionario para la Evaluación del Sindrome de Quemarse por el Trabajo - CESQT-PE - (Gil-Monte, 2005), adaptada para o Brasil por Gil-Monte, Carlotto e Câmara (2010). O instrumento é composto de 20 itens que se distribuem em quatro sub-escalas: Ilusão pelo trabalho (5 itens, alfa $=0,72$ ); Desgaste psíquico (4 itens, alfa $=0,86)$; Indolência (6 itens, alfa $=$ 0,75); e Culpa (5 itens, alfa $=0,79$ ); .3. Bateria de Avaliação de Riscos Psicossociais (UNIPSICO) de Gil-Monte (2005) que avalia: a) "Autonomia" (5 itens; alfa $=0,84)$, b) "Conflito de papel" (5 itens; alfa $=0,78), c)$ "Sobrecarga de trabalho" (6 itens; alfa $=0,79), d)$ "Apoio social no trabalho" (6 itens; alfa $=0,75)$, e) "Conflitos interpessoais" ( 6 itens; alfa $=0,76$ ). Todos os itens são avaliados com uma escala tipo Likert de frequência de cinco pontos (0 "Nunca" a 4 "Todos os dias").

\section{Procedimentos}

Para a coleta dos dados, primeiramente foi realizado um contato com a direção de cada instituição de ensino, sendo apresentado o objetivo do estudo, a fim de obter a autorização e o apoio para a aplicação dos instrumentos. Estes foram entregues pessoalmente aos professores, sendo a coleta realizada ao final da aplicação. Foi esclarecido aos professores tratar-se de uma pesquisa sem quaisquer efeitos avaliativos individuais e/ou institucional e que as respostas seriam anônimas e confidenciais. Foram realizados os procedimentos éticos conforme Resolução 466 do Conselho Nacional de Saúde do Brasil (CNS). A pesquisa possui aprovação do Comitê de Ética da Universidade Luterana do Brasil.

O Banco de Dados foi digitado e posteriormente analisado em pacote estatístico PASW, versão 17 (SPSS/PASW, Inc., Chicago, IL). Antes de realizar a análise de regressão linear múltipla (método stepwise), verificaram-se os pressupostos de multicolinearidade, normalidade, linearidade, homoscedasticidade e independência dos resíduos e outliers, sem identificar violação que contraindicasse sua utilização. A análise assumiu como variáveis dependentes as dimensões da SB (Ilusão para o trabalho, Desgaste psíquico, Indolência, e Culpa) e como variáveis independentes os riscos psicossociais (Autonomia, Conflito de papel, Sobrecarga de trabalho, Conflitos interpessoais e Apoio social). A seleção das variáveis preditoras adotou o nível de significância de $\mathrm{p}<0,05$. Na análise de regressão, o poder do efeito foi obtido pelos coeficientes de regressão padronizados calculados para cada modelo final (Field, 2009).

\section{Resultados}

Os resultados obtidos indicam que o maior índice médio foi obtido na variável Ilusão no trabalho e o menor na variável "Conflito de papel" (tabela 1). 
Tabela 1

Média e desvio-padrão, alfa e correlação entre as variáveis de estudo $(n=250)$

\begin{tabular}{|c|c|c|c|c|c|c|c|c|c|c|}
\hline Var. & M (DP) & $\alpha$ & 1 & 2 & 3 & 4 & 5 & 6 & 7 & 8 \\
\hline 1.IL & $3,18(0,68)$ & 0,74 & 1 & & & & & & & \\
\hline 2.DP & $1,85(0,78)$ & 0,76 & $-0,18^{* *}$ & 1 & & & & & & \\
\hline $3 . \mathrm{IN}$ & $0,91(0,58)$ & 0,76 & $-0,33^{* *}$ & $0,48^{* *}$ & 1 & & & & & \\
\hline 4.CL & $0,94(0,63)$ & 0,72 & $-0,19^{* *}$ & $0,40^{* *}$ & $0,56^{* *}$ & 1 & & & & \\
\hline 5.AU & $2,46(0,67)$ & 0,68 & $0,39^{* *}$ & $-0,01$ & $-0,06$ & $-0,04$ & 1 & & & \\
\hline 6.CP & $1,29(0,84)$ & 0,75 & $-0,15^{*}$ & $0,23^{* *}$ & $0,25^{* *}$ & $0,30^{* *}$ & $-0,38^{* *}$ & 1 & & \\
\hline 7.SL & $1,97(0,58)$ & 0,84 & $-0,11$ & $0,17^{* *}$ & $0,17^{* *}$ & $0,23^{* *}$ & $-0,19^{* *}$ & $0,63^{* *}$ & 1 & \\
\hline 8.AS & $2,37(0,87)$ & 0,73 & $0,40^{* *}$ & 0,05 & $-0,12$ & $-0,10$ & $0,27^{* *}$ & $-0,13$ & $-0,23^{* *}$ & 1 \\
\hline 9.CI & $1,75(0,74)$ & 0,87 & $-0,22^{* *}$ & $0,20^{*}$ & $0,54^{* *}$ & $0,54^{* *}$ & $-0,15$ & $0,47^{* *}$ & $0,26^{* *}$ & $-0,04$ \\
\hline
\end{tabular}

Nota: $* \mathrm{p}<0,05 ; *{ }_{\mathrm{p}}<0,01$; IL-Ilusão pelo trabalho; DP-Desgaste Psicológico; IN-Indolência; CL-Culpa; AU-Autonomia; CP-Conflito de Papel; SC-Sobrecarga Laboral; AS-Apoio Social; CI-Conflitos Interpessoais.

A tabela 2 apresenta os resultados da análise de regressão linear, que considerou as dimensões do Burnout como variáveis dependentes e, como variáveis preditoras, os riscos psicossociais. A análise dos preditores da dimensão de Ilusão pelo trabalho evidenciou um modelo explicativo constituído por 3 variáveis, que conjuntamente explicaram 39,3\% da variância desta dimensão, sendo "Autonomia" a variável de maior poder explicativo (31,9\%). O conjunto de variáveis revela que quanto menor a "Autonomia" e o "Apoio social", menor é o sentimento de Ilusão pelo trabalho e, quanto maior a presença de "Conflitos interpessoais" no contexto laboral, menor é a referida dimensão.

A variação da dimensão de Desgaste Psíquico é explicada somente pela variável "Conflito de papel" com $14,3 \%$ da variância. O resultado indica que quanto maior o "Conflito de papel" percebido pelo docente, maior é o sentimento de desgaste psicológico.

A Indolência também foi explicada por uma única variável: "Conflitos interpessoais" com um poder explicativo de $28,5 \%$, indicando que, quanto maior a presença de conflitos, maior é o comportamento de indiferença e distanciamento nas relações. Por fim, a análise de regressão para a variável dependente Culpa, revelou como variáveis preditoras, "Conflitos interpessoais" e "Conflito de papel", indicando que na medida em que aumenta a percepção de presença de conflitos interpessoais e de papel, aumenta o sentimento de culpa devido à percepção de que o docente não corresponde às expectativas sociais de seu papel.

Os resultados revelam um poder de efeito entre médio $\left(R^{2}=0,148\right)$ e elevado $\left(R^{2}=0,405\right)$, de acordo com os parâmetros recomendados por Field (2009). Nesse sentido, indica que as relações identificadas possivelmente também estarão presentes na população-alvo de professores universitários.

\section{Discussão}

Este estudo teve por objetivo avaliar os riscos psicossociais como variáveis preditoras das quatro dimensões da SB, a saber: Ilusão para o trabalho, Desgaste psíquico, Indolência e Culpa. Os resultados identificaram modelos explicativos compostos pelas variáveis "Autonomia", "Apoio social", "Conflitos interpessoais" e "Conflito de 
Tabela 2

Modelo da análise de regressão linear múltipla das dimensões de Burnout

\begin{tabular}{|c|c|c|c|c|c|c|c|}
\hline & $\mathrm{R}^{2}$ & $\mathrm{R}^{2 \text { Adjusted }}$ & $\mathrm{R}^{\text {change }}$ & B & SE & $\beta$ & $\mathrm{t}$ \\
\hline \multicolumn{8}{|c|}{ Ilusão pelo Trabalho } \\
\hline Autonomia & 0,319 & 0,314 & 0,319 & 0,469 & 0,066 & 0,470 & $7,079 * *$ \\
\hline Apoio social & 0,385 & 0,376 & 0,066 & 0,212 & 0,052 & 0,267 & $4,061 * *$ \\
\hline Conflitos interpessoais & 0,405 & 0,393 & 0,021 & $-0,133$ & 0,059 & $-0,145$ & $-2,269 *$ \\
\hline Model F & \multicolumn{7}{|c|}{$33,814^{* *}$} \\
\hline \multicolumn{8}{|c|}{ Desgaste Psíquico } \\
\hline Conflito de papel & 0,148 & 0,143 & 0,148 & 0,319 & 0,062 & 0,385 & $3,125^{* *}$ \\
\hline Model F & \multicolumn{7}{|c|}{$26,267 * *$} \\
\hline \multicolumn{8}{|c|}{ Indolência } \\
\hline Conflitos interpessoais & 0,290 & 0,285 & 0,290 & 0,390 & 0,050 & 0,538 & $7,845^{* *}$ \\
\hline \multicolumn{8}{|c|}{ Model F $61,543^{* *}$} \\
\hline \multicolumn{8}{|c|}{ Culpa } \\
\hline Conflitos interpessoais & 0,292 & 0,287 & 0,292 & 0,337 & 0,065 & 0,378 & $5,198 * *$ \\
\hline Conflito de papel & 0,346 & 0,376 & 0,092 & 0,318 & 0,067 & 0,345 & $4,743 * *$ \\
\hline \multicolumn{8}{|c|}{ Model F 46,742** } \\
\hline
\end{tabular}

Nota: $* \mathrm{p}<0,05, * * \mathrm{p}<0,01$.

papel". A análise dos preditores da dimensão de Ilusão pelo trabalho revelou que quanto maior a “Autonomia" e o "Apoio social" maior é a Ilusão pelo trabalho. Quanto maior a presença de "Conflitos interpessoais" no contexto laboral, menor é o sentimento de entusiasmo no trabalho. Estudo realizado por Carlotto (2004) com professores universitários identificou associação positiva entre a autonomia e realização no trabalho.

O resultado referente à relação entre "Autonomia" e "Ilusão pelo trabalho", considerando que se trata da busca do professor para alcançar suas metas laborais e avaliar sua atividade como atrativa e fonte de realização pessoal, pode ser entendido a partir do modelo motivacional de Hackman e Oldham (1980). Nesse, a "Autonomia" é uma característica básica de um cargo que gera uma maior percepção de responsabilidade quanto ao resultado do seu trabalho, o que implicaria em maior motivação intrínseca. De acordo com Adebayo e Ezeanya (2011), a "Autonomia" pode ser um recurso necessário para alcançar as metas de trabalho.

Quanto ao "Apoio social”, resultado semelhante foi identificado por Salami (2011). Para o autor, quando os professores recebem "Apoio social" de seus supervisores e colegas para enfrentar as dificuldades no trabalho, isso ajuda a minimizar o sofrimento emocional e aumentar a sua autoestima, implicando em uma melhora de suas habilidades em lidar de forma eficaz com os problemas cotidianos do trabalho. Essa melhora na forma de resolver os problemas relacionados ao estresse possibilita o alcance das metas e o sentimento de realização profissional.

Uma das competências comportamentais exigidas do professor universitário é a interpessoal, em nível individual e grupal, o que envolve capacidade 
de desenvolver e manter empatia (Mendonça et al., 2012). Assim, na medida em que o professor percebe estar trabalhando em um contexto de "Conflitos interpessoais", seja com alunos, colegas ou gestores, pode entender que estes sejam uma barreira para atingir suas metas profissionais, o que explicaria a relação entre "Conflitos interpessoais" e menor Ilusão pelo trabalho.

O Desgaste psíquico teve como preditora a variável "Conflito de papel", indicando que sua elevação ocasiona maior sentimento de desgaste, confirmando estudos realizados por Jawahar, Stone \& Kisamore (2007), Olivares-Faúndez, Gil-Monte, Mena, Jélvez-Wilke \& Figueiredo-Ferraz (2014). O "Conflito de papel" ocorre quando o indivíduo recebe mensagens para executar dois ou mais papéis incompatíveis ou conflitantes (Jones, 1993). $\mathrm{O}$ "Conflito de papel" pode estar relacionado às demandas do cargo ou entre valores importantes (Maslach \& Goldberg, 1998). Uma das mais frequentes formas de "Conflito de papel" é a sobrecarga, ou seja, ocorre conflito quando o indivíduo não consegue executar suas atividades dentro do tempo e com os recursos disponíveis. Essa deve ser diferenciada entre sobrecarga qualitativa (tarefas difíceis de serem realizadas) e sobrecarga quantitativa (muitas tarefas a serem realizadas) (Kahn, 1978).

Pode-se pensar que no caso da docência em nível universitário as duas situações podem estar presentes: alta demanda quantitativa e qualitativa e conflito entre valores pessoais e institucionais. Nos últimos anos, as universidades têm, cada vez mais, realizando movimentos para ajustar-se às exigências de um mercado altamente competitivo, principalmente nas universidades privadas. Assim, uma nova organização do trabalho é imposta ao docente que tem sua eficiência e produtividade objetivadas em índices (Mancebo, 2007). Pode-se pensar que o docente, ao ver seu trabalho materializado, entra em conflito com o que intrinsicamente caracteriza o trabalho docente, ou seja, um trabalho imaterial (Borsoi, 2012). O trabalho docente se organiza em termos de sua imaterialidade, caracterizado fundamentalmente pelo viés intelectual e relacional. Para atender às exigências voltadas para a subjetividade, prazer e as demandas institucionais, o profissional sobrecarrega-se quanti e qualitativamente.

As mudanças ocorridas na universidade e a submissão aos valores de mercado se traduzem em uma perda da identidade docente, o que resulta em contradições entre as funções tradicionais, as novas e diversificadas funções que são atribuídas ao professor. As novas exigências e responsabilidades podem ocasionar não somente um aumento da carga de trabalho, em extensão e intensidade, mas também podem se constituir em uma fonte de conflito com as atividades acadêmicas, de fragmentação do tempo de trabalho e de dispersão de energia (Silva, 2011). Os problemas de desempenho em universidades, relacionados ao estresse, são muitas vezes tolerados pela cultura acadêmica e os gestores frequentemente relutam em lidar com os sintomas observados e questões comportamentais associadas à SB docente (Minter, 2009).

Quanto à Indolência, essa foi explicada pelo aumento dos "Conflitos interpessoais" no contexto de trabalho. Resultado semelhante foi identificado em estudo realizado por Malmann, Palazzo, Carlotto \& Aerts (2009). Pode-se pensar que, na medida em que o docente desenvolve suas atividades e estas incluem conflitos nas relações, ele tende a proteger-se do desgaste ocasionado e adota atitudes de indiferença e distanciamento das pessoas no contexto de trabalho. Tal comportamento pode funcionar como uma estratégia defensiva visando à manutenção da energia e recursos necessários para atender as demandas e manter-se ativo no trabalho (Maslach et al., 2001).

A dimensão de Culpa revelou como variáveis preditoras os "Conflitos interpessoais" e o "Conflito de papel". Esse resultado evidencia que o aumento da percepção de conflitos interpessoais e de papel 
implica em uma elevação no sentimento de culpa relacionado à percepção de que o docente não corresponde às expectativas sociais de seu papel.

$\mathrm{Na}$ atualidade, a universidade possui interesses antagônicos. Os governos esperam uma universidade a serviço do desenvolvimento. Os alunos desejam formação profissional voltada para o desenvolvimento de habilidades que lhes permitam a empregabilidade e competitividade no mundo do trabalho, ou seja, a universidade funcional. Os professores desejam produzir conhecimento, fazer ciência com condições objetivas favoráveis, isto é, a universidade clássica ou do conhecimento. Por outro lado, o mercado deseja inovação e tecnologia a serviço da produção, profissionais formados dentro de expectativas econômicas, ou seja, uma universidade de resultados (Silva, 2011). De acordo com a autora, esses desafios e expectativas postos à universidade influenciam sua organização, estrutura, suas dinâmicas de trabalho e o trabalho acadêmico. Esse processo é marcado por tensões e contradições que afetam a atividade profissional dos professores universitários. Diante da dificuldade em acompanhar esta nova configuração do trabalho docente em um curto espaço de tempo, pode-se considerar que o sentimento de culpa decorrente do seu papel profissional neste contexto seja uma possibilidade.

Uma das forças do presente estudo é que os dados foram coletados entre professores de universidades particulares, o que reduz o viés associado a professores de instituições públicas, que possuem especificidades como isonomia estrutural e de remuneração entre professores, plano de carreira vinculado ao Ministério da Educação e estabilidade laboral (Aquino, Moita, Correa, \& Souza, 2014). Outra diz respeito à utilização de um consistente modelo teórico e instrumento adaptado para o contexto brasileiro com adequados índices de confiabilidade. O tamanho da amostra mostrou-se suficiente para revelar bons níveis de poder de efeito para a análise estatística realizada.
O estudo, todavia, apresenta algumas limitações que devem ser consideradas na leitura de seus resultados. Uma delas é seu delineamento transversal que impede a análise de relações causais. Outra é a utilização de medidas de autorrelato que pode gerar um viés relacionado à desejabilidade social. Especificamente neste estudo, podem-se citar as questões relacionadas à dimensão de Indolência, pois pode ser difícil para o professor assumir que se distancia e trata de forma impessoal as pessoas que atende.

Por fim, pode-se pensar na regionalidade da amostra investigada, a qual possui características distintas de outras regiões do país. Esta situação é importante, uma vez que hoje já está clara a influência de aspectos culturais e contexto laboral sobre a SB. Sarason (1985) afirma que tanto os valores sociais, como as condições econômicas e os momentos históricos, são elementos decisivos para explicar os processos de desenvolvimento individuais e coletivo da SB, tendo em vista que ele reflete as amplas estruturas de uma determinada sociedade.

Os resultados obtidos sugerem a necessidade de aprofundamento por meio de estudos longitudinais, com amostras de diferentes estados e regiões do Brasil. Em relação às implicações para a prática, sugerem-se ações voltadas para a reconfiguração do conteúdo do cargo e atividades docentes. Tais medidas visariam assegurar maior autonomia e maior aproximação entre metas individuais e organizacionais no trabalho. Da mesma forma propõem-se ações nas relações de trabalho, no sentido de maior sensibilização quanto à importância do apoio social e da qualidade das relações no trabalho.

\section{Referências}

Adebayo, S. O., \& Ezeanya, O. D. (2011). Task identity and job autonomy as correlates of burnout among nurses in Jos, Nigeria. International 
Review of Social Sciences and Humanities, 2(1), 7-13.

Aquino, C. A. B. de, Moita, D. S., Correa, G. M., \& Oliveira Souza, K.O. (2014). O fenômeno da precarização e da flexibilização laboral no âmbito da universidade pública brasileira: o caso dos professores substitutos. Athenea Digital, 14(1), 173-193.

Barkhuizen, N., \& S. Rothmann (2008). Occupational stress of academic staff in South African higher education institutions. South African Journal of Psychology, 38(2), 321-336. doi: $10.1177 / 008124630803800205$

Borsoi, I. C. F. (2012). Trabalho e produtivismo: saúde e modo de vida de docentes de instituições públicas de Ensino Superior. Cadernos de Psicologia Social do Trabalho, 15(1), 81-100.

Carlotto, M. S. (2004). Síndrome de burnout e características de cargo em professores universitários. Revista Psicologia: Organizações e Trabalho, 4(2), 145-162.

Catano, V., Francis, L., Haines, T., Kirpalani, H., Shannon, H., Stringer, B., \& Lozanzki, L. (2010). Occupational stress in Canadian universities: a national survey. International Journal of Stress Management, 17(3), 232-258. doi:10.1037/ a0018582

Cooper, C. L., Dewe, P. J., \& O’Driscoll, M. P. (2001). Organizational stress: A review and critique of theory, research, and applications. Thousand Oaks, CA: Sage doi:10.1093/ bjsw/32.5.648

Droogenbroeck, F. V., Spruyt, B., \& Vanroelen, C. (2014). Burnout among senior teachers: investigating the role of workload and interpersonal relationships at work. Teaching and Teacher Education, 43, 99-109. doi:10.1016/j. tate.2014.07.005

Farber, B. A. (1991). Crisis in education. Stress and burnout in the American teacher. São Francisco: Jossey-Bass Inc.

Field, A. (2009). Discovering statistics with SPSS ( $\left.2^{\text {nd }} e d.\right)$. London: Sage Publications.
Franco, M. E. D. P. (2001). Comunidade de conhecimento, pesquisa e formação do professor do ensino superior. In M. C. Morosini (Org.), Docência universitária e os desafios da realidade nacional (pp.109-135). Brasília: Plano.

Gillespie, N. A., Walsh, M., Winefield, A. H., Dua, J., $\&$ Stough, C. (2001). Occupational stress in universities: staff perceptions of the causes, consequences and moderators of stress. Work \& Stress, 15(1), 53-72. doi: 10.1080/02678370117944

Gil-Monte, P. R. (2005). El sindrome de quemarse por el trabajo ("burnout"). Una enfermedad laboral en la sociedad del bienestar. Madrid: Pirámide.

Gil-Monte, P. R. (2008). El síndrome de quemarse por el trabajo (burnout) como fenómeno transcultural. Información Psicológica, 91-92, 4-11.

Gil-Monte, P. R., Carlotto, M. S., \& Câmara, S. G. (2010). Validation of the Brazilian version of the "Spanish Burnout Inventory" in teachers. Revista de Saúde Pública, 44(1), 140-147.

Guglielmi, R. S., \& Tatrow, K. (1998). Occupational stress, burnout, and health in teachers: a methodological and theoretical analysis. Review of Educational Research, 68(1), 61-69. doi: 10.3102/00346543068001061

Gupta, M., \& Rani, S. (2014). Burnout: a serious problem prevalent among teachers in the present times. Journal Of Education \& Research, 4(1). Disponível em http://gangainstituteofeducation. com/NewDocs/2.pdf

Hackman, J. R., \& Oldhan, G. R. (1980). Work redesign. Massachustts: Addison - Wesley Reading.

Jawahar, I. M., Stone, T. H., \& Kisamore, J. L. (2007). Role conflict and burnout: the direct and moderating effects of political skill and perceived organizational support on burnout dimensions. International Journal of Stress Management, 14(2), 142-159. doi:10.1037/10725245.14.2.142

Jones, M. L. (1993). Role conflict: causes of burnout or energizer. Social Work, 38(2), 136-142. doi: $10.1093 / \mathrm{sw} / 38.2 .136$ 
Kahn, R. (1978). Job burnout-prevention and remedies. Public Welfare, 36(2), 61-63.

Kang, L. S., \& Sidhu, H. (2015). Identification of stressors at work: a study of university teachers in India. Global Business Review, 16(2), 303320. doi: 10.1177/0972150914564421

Leiter, M. P., Bakker, A. B., \& Maslach, C. (2014). Burnout at work. New York: Taylor \& Francis.

Leka, S., Griffiths, A., \& Cox, T. (2003). Work organisation and stress. Geneva: World Health Organization.

Mallmann, C. S., Palazzo, L. S., Carlotto, M. S., \& Aerts, D. R. G. de C. (2009). Fatores associados à síndrome de burnout em funcionários públicos municipais. Psicologia: Teoria e Prática, 11(2), 69-82.

Mancebo, D. (2007). Trabalho docente: subjetividade, sobre implicação e prazer. Psicologia: Reflexão e Crítica, 20(1), 74-80. doi:10.1590/ S0102-79722007000100010

Maslach, C., \& Goldberg, J. (1998). Prevention of burnout: news perspectives. Applied \& Preventive Psychology, 7, 63-74. doi:10.1016/S09621849(98)80022-X

Maslach, C., Schaufeli, W. B., \& Leiter, M. P. (2001). Job burnout. Annual Review Psychology, 52(1), 397-422. doi: 10.1146/annurev.psych.52.1.397

Mendonça, J. R. C., Paiva, K. C. M., Padilha, M. A. S., \& Barbosa, M. A. C. (2012). Competências profissionais de professores do ensino superior no Brasil: proposta de um modelo integrado. In Anais da II Conferência do Forges - Fórum da Gestão do Ensino Superior nos Países e Regiões de Língua Portuguesa (p.), Macau, China. Disponível em http://aforges.org/conferencia2/docs_documentos/Paralela_3/Mendonca_Jose_et_al_(Brasil).pdf

Minter, R. L. (2009). Faculty burnout. Contemporary Issues In Education Research, 2(2). Disponível em http://www.cluteinstitute.com/ojs/index. $\mathrm{php/CIER/article/view/1090/1074}$

Moreno-Jiménez, B., Garrosa, E., Rodríguez, R., Martínez, M., \& Ferrer, R. (2010). El burnout del profesorado universitario y las intenciones de abandono: un estudio multi-muestra. Revista de Psicología del Trabajo y de las Organizaciones, 25(2), 149-163.

Norlund, S., Reuterwall, C., Höög, J., Janlert, U., \& L. S Järvholm (2015). Work situation and self-perceived economic situation as predictors of change in burnout-a prospective general population-based cohort study. BMC Public Health, 15, 329. Disponível em http://www.biomedcentral.com/1471-2458/15/329. doi:10.1186/ s12889-015-1681-x

Olivares-Faúndez, V. E., Gil-Monte, P. R., Mena, L., Jélvez-Wilke, C., \& Figueiredo-Ferraz, H. (2014). Relationships between burnout and role ambiguity, role conflict and employee absenteeism among health workers. Terapia Psicológica, 32(2), 111-120. doi: 10.4067/S071848082014000200004

Papastylianou, A., Kaila, M., \& Polychronopoulos, M. (2009). Teachers' burnout, depression, role ambiguity and conflict. Social Psychology of Education: an International Journal, 12(3), 295-314. doi: 10.1007/s11218-008-9086-7

Salami, S. O. (2011). Job stress and burnout among lecturers: personality and social support as moderators. Asian Social Science, 7(5), 110-121. doi: 10.5539/ass.v7n5p110

Sarason, S. B. (1985). Caring and compassion in clinical practice. San Francisco: Jossey Bass.

Silva, C. (2011). Ser professor universitário em tempos de mudança: a profissão acadêmica e suas reconfigurações. (Tese de Doutoramento em Educação - Administração e Política Educacional. Universidade de Lisboa - Instituto de Educação, Portugal).

Tytherleigh, M. Y., Webb, C., Cooper, C. L., \& Ricketts, C. (2005). Occupational stress in UK higher education institutions: a comparative study of all staff categories. Higher Education Research and Development, 24(1), 41-61. doi:10.1080/0729436052000318569 
Tümkaya, S. (2006). Faculty burnout in relation to work environment and humor as a coping strategy. Educational Sciences: Theory \& Practice, 6(3), 911-921.

Yong, Z., \& Yue, Y. (2007). Causes for burnout among secondary and elementary school teachers and preventive strategies. Chinese Education and Society, 40(5), 78-85. doi:10.2753/CED10611932400508

Recebido: Julho 01, 2015

Aprovado: Agosto 02, 2016 
